Übertragungsrisiko gesenkt

\section{Schwangere mit HIV sind gut versorgt}

In Deutschland hat man es geschafft, die Mutter-Kind-Übertragungsrate des AIDS-Erregers sehr niedrig zu halten.

Als eine medikamentöse Transmissionsprophylaxe noch nicht möglich war, ließ sich in vielen Fällen bei HIV-infizierten Schwangeren die Übertragung des AIDS-Erregers auf den Nachwuchs nicht verhindern. Die Übertragungsrate lag damals zwischen 15 und 30\%. Das hat sich drastisch geändert. Heute geht man davon aus, dass die Übertragungsrate mithilfe einer antiretroviralen Kombinationstherapie nur noch unter $1 \%$ liegt.

\section{Behandlungsindikationen}

Wie es in der deutsch-österreichischen Leitlinie zur HIV-Therapie in der Schwangerschaft von 2014 heißt, gelten für HIV-positive Schwangere die gleichen Behandlungsindikationen wie für nichtschwangere Erwachsene. Das bedeutet, dass jede symptomatische HIV-Infektion eine Indikation zur Therapie ist. Bei asymptomatischem Verlauf der Infektion liege spätestens ab einer CD4-Zellzahl

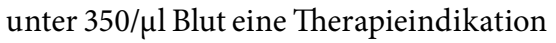
vor, so die Empfehlung. Unter anderem kann eine chronische Hepatitis Anlass sein, die Therapie bereits bei höheren Zellzahlen zu beginnen.

Unbedingt zu berücksichtigen ist der Leitlinie zufolge die Tatsache, dass auch bei gesunden Frauen während der Schwangerschaft die Zahl der CD4-positiven Lymphozyten um 10 bis $20 \%$ fallen kann. Sind Schwangere HIV-infiziert, kann der Abfall bis zu 40\% betragen.

Für die Initialtherapie bei einer Infektion der Mutter wird eine Kombinationsbehandlung aus zwei nukleosidischen Reverse-Transkriptase-Hemmern (NRTI) plus einem geboosterten Proteasehemmer oder zwei NRTIs plus einem nicht-nukleosidischen Reverse-Transkriptase-Hemmer empfohlen. Weil sich die Pharmakokinetik während der Schwangerschaft verändert, können Medikamentenspiegelmessungen unter Umständen sinnvoll sein. Laut den Angaben des Robert-KochInstituts in Berlin wurden im Jahr 2014 in Deutschland nur 25 Mutter-Kind-Übertragungen gemeldet. Ein Jahr zuvor waren es sogar nur 21 Übertragungen.

(ple)

\title{
Bericht von UNAIDS
}

\section{Immer mehr HIV-Infizierte behandelt}

Um die AIDS-Pandemie bis $2030 \mathrm{zu}$ beenden, muss sich die Versorgung von HIV-Patienten weiter verbessern.

Das Ziel der Organisation UNAIDS ist es, die AIDS-Pandemie bis zum Jahr 2030 zu beenden. Dazu gehört ein wichtiges Teilziel, nämlich im Jahr 2020 insgesamt $90 \%$ derjenigen, die von ihrer Infektion wissen, mit einer antiretroviralen Therapie (ART) versorgen zu können. Im Jahr 2015 erhielten bereits 17 Millionen Menschen eine ART - das sind zwei Millionen Menschen mehr als ein Jahr zuvor, wie UNAIDS jetzt berichtet. Weltweit waren 2015 etwa 36 Millionen Men- schen mit dem AIDS-Erreger infiziert, ungefähr zwei Millionen hatten sich in dem Jahr mit dem Virus angesteckt. Etwa zwei Drittel der Neuinfektionen wurden aus Ländern südlich der Sahara gemeldet.

\section{HIV in Deutschland}

In Deutschland leben derzeit etwa 83.400 Menschen mit einer HIV-Infektion oder sind bereits an AIDS erkrankt. Nach Angaben des Robert-Koch-Instituts wissen ungefähr 13.200 Menschen nichts von ihrer Infektion. Es wird geschätzt, dass in Deutschland etwa $80 \%$ aller Betroffenen eine ART erhalten.

\section{ÜBRIGENS}

... altern chronisch mit HIV infizierte Menschen schneller. Epigenetisch betrachtet sind sie demnach fünf Jahre älter als Gesunde im gleichen Alter, hat eine aktuelle USamerikanische Studie ergeben.

Molecular Cell. 2016: 62; 157-168.

... wird Prof. Dr. Michel C. Nussenzweig von der Rockefeller University in New York für Arbeiten zu breit neutralisierenden Antikörpern gegen HIV mit dem RobertKoch-Preis geehrt.

...liefern evolutionsbiologische, auf der CROI-Tagung 2016 in Boston, Massachusetts, präsentierte Daten zu HIV nun den Beleg dafür, dass der Frankokanadier Gaëtan Dugas nicht der vermeintliche Indexpatient für die USA sein kann.

CROI 2016, Boston, Februar 2016,

Abstract Nummer 140.

Punkte sammeln in der

Sommerakademie mit e.Med

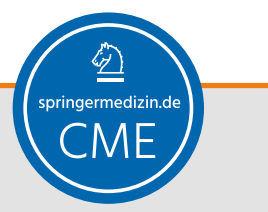

Auch 2016 haben wir in der Sommerakademie ausgewählte CME-Kurse für Ihre hausärztliche Fortbildung zusammengestellt.

Zum Thema "Aids/HIV“ finden Sie unter www.springermedizin.de/sommerakademie aktuell den Kurs

„Update: HIV-Infektionen:

AIDS - früh erkannt, Gefahr gebannt!"

Zur Teilnahme benötigen Sie das e.Med-Abo, mit dem Sie Zugang zu allen Inhalten auf Springer-Medizin.de erhalten - kombiniert mit einer gedruckten Fachzeitschrift Ihrer Wahl. Als e.Med-Abonnent haben Sie nicht nur Zugriff auf die Sommerakademie, Sie können das komplette CME-Kursangebot von SpringerMedizin.de nutzen. Hier finden Sie aktuell rund 550 CME-zertifizierte Fortbildungskurse aus allen medizinischen Fachrichtungen! Damit ist CME.SpringerMedizin.de Deutschlands erste Adresse für Online-CMEFortbildung.

Nutzen Sie die Sommerzeit, um CME-Punkte zu sammeln und um sich schnell und effizient fortzubilden. Testen Sie CME.SpringerMedizin.de 30 Tage lang kostenlos und unverbindlich mit dem e.Med-Abo: www.springermedizin.de/eMed 